$\underline{\text { Preprint typeset in JHEP style - HYPER VERSION }}$

IFUM-1002-FT

LTH 962

\title{
Nonextremal black holes in gauged supergravity and the real formulation of special geometry II
}

\author{
Dietmar Klemm $^{a b}$ and Owen Vaughan ${ }^{c}$ \\ ${ }^{a}$ Dipartimento di Fisica, Università di Milano, \\ Via Celoria 16, 20133 Milano, Italy. \\ ${ }^{b}$ INFN, Sezione di Milano, Via Celoria 16, 20133 Milano, Italy. \\ ${ }^{c}$ Department of Mathematical Sciences, \\ University of Liverpool, Peach Street, Liverpool L69 7ZL, UK.
}

\begin{abstract}
In arXiv:1207.2679 a new prescription for finding nonextremal black hole solutions to $\mathcal{N}=2, D=4$ Fayet-Iliopoulos gauged supergravity was presented, and explicit solutions of various models containing one vector multiplet were constructed. Here we use the same method to find new nonextremal black holes to more complicated models. We also provide a general recipe to construct non-BPS extremal solutions for an arbitrary prepotential, as long as an axion-free condition holds. These follow from a set of first-order conditions, and are related to the corresponding supersymmetric black holes by a multiplication of the charge vector with a constant field rotation matrix $S$. The fake superpotential driving this first-order flow is nothing else than Hamilton's characteristic function in a Hamilton-Jacobi formalism, and coincides in the supersymmetric case (when $S$ is plus or minus the identity) with the superpotential proposed by Dall'Agata and Gnecchi in arXiv:1012.3756. For the nonextremal black holes that asymptote to (magnetic) AdS, we compute both the mass coming from holographic renormalization and the one appearing in the superalgebra. The latter correctly vanishes in the BPS case, but also for certain values of the parameters that do not correspond to any known supersymmetric solution of $\mathcal{N}=2$ gauged supergravity. We finally show that the product of all horizon areas depends only on the charges and the asymptotic value of the cosmological constant.
\end{abstract}


KeYwords: Black Holes in String Theory, AdS-CFT Correspondence, Superstring Vacua. 


\section{Contents}

1. Introduction 1

2. Review of method 3

2.1 Equations of motion for generic models

2.2 Metric ansatz 6

3. The $F(X)=-2 i\left(X^{0} X^{1} X^{2} X^{3}\right)^{1 / 2}$ model 7

3.1 Equations of motion 7

3.2 Nonextremal black holes 9

3.3 First-order equations and fake superpotential

3.4 Recovering the four-charge solution of $\mathcal{N}=8$ gauged supergravity

4. The $F(X)=-X^{1} X^{2} X^{3} / X^{0}$ model

4.1 Equations of motion

4.2 First-order equations and fake superpotential 19

5. Fake superpotential for arbitrary prepotential 22

6. Conclusions and final remarks 26

A. Hesse potential for $F=-2 i\left(Y^{0} Y^{1} Y^{2} Y^{3}\right)^{1 / 2}$ model 27

\section{Introduction}

Black holes in anti-de Sitter (AdS) spaces play an important role in the AdS/CFT correspondence. In the BPS case, for instance, their conformal boundaries can provide curved backgrounds on which supersymmetric field theories can be defined $[1,2]$. On the other hand, nonextremal black holes are instrumental in recent AdS/CFT applications to condensed matter physics, since they are dual to certain condensed matter systems at finite temperature. A basic ingredient of realistic condensed matter systems is the presence of a finite density of charge carriers, which implies the necessity of a bulk 
$\mathrm{U}(1)$ gauge field. A further step in modeling strongly coupled holographic systems is to include the leading relevant (scalar) operator in the dynamics. This is generically uncharged, and is dual to a neutral scalar field in the bulk. One is thus naturally led to consider nonextremal charged black holes in gauged supergravity with running scalars.

AdS can be viewed as a deformation of Minkowski spacetime, and one would like to understand how much of the features of asymptotically flat black holes carry over to their cousins in AdS, or, more generally, how certain recipes to construct such solutions get modified when one turns on a cosmological constant or a potential for the moduli. For example, it is known how to go from vanishing to nonzero temperature for asymptotically flat black holes and black branes, namely by introducing a so-called blackening function in certain components of the metric. Although something similar seems to work in special cases [3], the AdS analogue of this procedure is unknown in general.

Recently there has also been an increasing interest in the attractor mechanism in presence of a scalar potential [4-8]. Unless the latter has flat directions, the values of the moduli at infinity will be completely fixed, and can thus not be continuously varied. It is therefore clear that the attractor mechanism in this case will be qualitatively different from the one for asymptotically flat black holes. In fact, new properties emerge in gauged supergravity, for instance possible nontrivial moduli spaces for the BPS attractor flow [5]. This is in contrast to ungauged supergravity, where there are no flat directions in the black hole potential for BPS flows [9] (at least as long as the metric of the scalar manifold is strictly positive definite), but there is a nontrivial moduli space for non-BPS flows $[10,11]$.

A further point that motivates studying AdS black holes is the existence of solitons, i.e., there are solutions that have no smooth limit when the coupling constant goes to zero, like Romans' cosmic monopole [12] and its generalizations [5, 13].

For these reasons, it would be desirable to dispose of a more complete picture of possible asymptotically AdS black holes, at least for supergravity models that can be consistently embedded in string theory. One such model is four-dimensional $\mathcal{N}=2$ gauged supergravity with prepotential $F=-2 i\left(X^{0} X^{1} X^{2} X^{3}\right)^{1 / 2}$, that will be considered below. Known black hole solutions to this theory, which contains three vector multiplets, include the static four-charge black holes of [14] and the rotating ones of [15], that have two pairwise equal electric charges. However, the most general rotating solution with nut-charge, four electric and four magnetic charges, containing also the BPS black holes of $[5,16,17]$, has not been constructed so far $^{1}$.

\footnotetext{
${ }^{1}$ The supersymmetric solutions of [17], which were found by using the general recipe provided in [18], contain two pairwise equal electric and magnetic charges as well as nut charge. These are not all independent, but are given in terms of three free parameters.
} 
In [19] a new prescription for finding nonextremal black hole solutions to $\mathcal{N}=2$, $D=4$ Fayet-Iliopoulos gauged supergravity was presented, and explicit solutions of various models containing one vector multiplet were constructed. Here we shall go one step further, and use the same method to find new nonextremal black holes to more complicated models.

The remainder of this paper is organized as follows: In section 2 we review the recipe of [19], which made essential use of the formalism developed in [20], based on dimensional reduction and the real formulation of special geometry. In section 3, the $F=-2 i\left(X^{0} X^{1} X^{2} X^{3}\right)^{1 / 2}$ model is considered, and new nonextremal black holes are constructed. They carry four magnetic charges, and contain both the solutions of [14] and the supersymmetric ones found in [5]. We compute their entropy, temperature, the mass coming from holographic renormalization and the one appearing in the superalgebra. The latter correctly vanishes in the BPS case, but quite surprisingly also for certain values of the parameters that do not correspond to any known supersymmetric solution of $\mathcal{N}=2$ gauged supergravity. It is moreover shown that the product of all horizon areas depends only on the charges and the asymptotic value of the cosmological constant, confirming the universality of the area-product formula [21]. In the following section, we consider the model with prepotential $F=-X^{1} X^{2} X^{3} / X^{0}$. In the ungauged case, this is related to the one of section 3 by a symplectic transformation, but since the gauging breaks symplectic covariance, this will lead to new physics. In fact, unlike the solutions of 3, those that we shall construct in 1 do not asymptote to AdS.

For both models, we find that an extremal subclass of these solutions ${ }^{2}$, which is related to the corresponding supersymmetric black holes by a simple individual signflipping of the charges, follows from a set of first-order conditions. The fake superpotential appearing in these first-order equations is nothing else than Hamilton's characteristic function in a Hamilton-Jacobi formalism. It is shown in section 5 that this can be generalized to any prepotential, as long as a zero-axion condition holds. This provides a general recipe that allows to construct non-BPS extremal black holes in $\mathcal{N}=2$ FI-gauged supergravity. We obtain the fake superpotential $W$, and show that in the supersymmetric case (when the so-called field rotation matrix is plus or minus the identity), $W$ coincides with the true superpotential proposed by Dall'Agata and Gnecchi in [6].

We conclude in 6 with an outlook and some final remarks.

\section{Review of method}

In this section we review the prescription for finding nonextremal solutions presented

\footnotetext{
${ }^{2}$ Actually, for the $F=-X^{1} X^{2} X^{3} / X^{0}$ model, we only find extremal solutions.
} 
in [19], which is based on dimensional reduction over the timelike dimension and the formulation of projective special Kähler geometry in terms of real rather than complex coordinates. The final ingredient is a coordinate redefinition that allows the moduli fields to absorb the Kaluza-Klein scalar (a.k.a. the metric warp factor) in order to lift a hypersurface constraint. For more information on this procedure we refer the reader to [20], in which this approach was developed for four-dimensional $\mathcal{N}=2$ (ungauged) supergravity for the first time, and new rotating and static solutions to the ungauged theory were discussed.

This approach was originally developed in [22] for five-dimensional supergravity. In the same paper new multi-centered extremal solutions were found, which have subsequently been generalised to non-extremal solutions for certain classes of models [23,24]. When restricted to to static and spherically symmetric backgrounds the equations of motion also take a similar in form to those recently presented in [25].

\subsection{Equations of motion for generic models}

Our starting point is the Lagrangian given by expression (2.7) of [19]:

$$
\mathcal{L}_{3} \sim-\frac{1}{2} R_{3}-\tilde{H}_{a b}\left(\partial_{\mu} q^{a} \partial^{\mu} q^{b}-\partial_{\mu} \hat{q}^{a} \partial^{\mu} \hat{q}^{b}\right)+\frac{1}{2 H} V
$$

which is a three-dimensional effective Lagrangian for a theory of Fayet-Iliopoulos gauged supergravity coupled to $n$ vector multiplets on static backgrounds. It is understood that the four-dimensional spacetime metric has been decomposed in the standard manner adapted to dimensional reduction,

$$
d s_{4}^{2}=-e^{\phi}\left(d t^{2}+V_{\mu} d x^{\mu}\right)+e^{-\phi} d s_{3}^{2} .
$$

We refer to $e^{\phi}$ as the Kaluza-Klein scalar and $V_{\mu}$ as the Kaluza-Klein vector. The latter vanishes for static backgrounds when appropriate choices of coordinates are made, which we will assume from now on. The scalar fields $q^{a}=\left(x^{I}, y_{I}\right)^{T}$ appearing in (2.1) represent the combined degrees of freedom of both the complex scalar fields $X^{I}, \bar{X}^{I}$ and the KK-scalar $e^{\phi}$,

$$
\begin{aligned}
x^{I} & :=\operatorname{Re} Y^{I}=e^{\phi / 2} \operatorname{Re} X^{I}, \\
y_{I} & :=\operatorname{Re} F_{I}(Y)=e^{\phi / 2} \operatorname{Re} F_{I}(X) .
\end{aligned}
$$

Here we have implicitly defined $Y^{I}, F_{I}$ as rescaled versions of $X^{I}, F_{I}$. The scalar fields $\hat{q}^{a}=\left(\frac{1}{2} \zeta^{I}, \frac{1}{2} \tilde{\zeta}_{I}\right)^{T}$ descend from the degrees of freedom of the gauge fields,

$$
\begin{aligned}
& \partial_{\mu} \zeta^{I}:=F_{\mu 0}^{I}, \\
& \partial_{\mu} \tilde{\zeta}_{I}:=G_{I \mid \mu 0} .
\end{aligned}
$$


The scalar couplings are completely determined by the Hesse potential $H(x, y)$, which plays the role of the holomorphic prepotential $F(X)$ when using real coordinates. The two potentials are related by a Legendre transformation that replaces $\operatorname{Im}\left(X^{I}\right)$ with $\operatorname{Re}\left(F_{I}\right)$ as an independent field [26]. The Hesse potential also happens to be proportional to the Kähler potential ${ }^{3}$. The coupling matrix that appears in (2.1) is given in terms of the Hesse potential by

$$
\tilde{H}_{a b}=\frac{\partial^{2} \tilde{H}}{\partial q^{a} \partial q^{b}}
$$

where $\tilde{H}=-\frac{1}{2} \log -2 H$. The function $V$ appearing in the Lagrangian is nothing other than the Fayet-Iliopoulos potential, and the whole term $\frac{1}{H} V$ can be written in terms of the Hesse potential as

$$
\frac{1}{H} V(q)=2 g_{I} g_{J}\left(-\frac{\partial^{2}}{\partial y_{I} \partial y_{J}} \tilde{H}+\frac{2}{H^{2}} x^{I} x^{J}+4 \frac{\partial \tilde{H}}{\partial y_{I}} \frac{\partial \tilde{H}}{\partial y_{J}}\right)
$$

This simple expression for this potential is another major advantage of using real coordinates and their associated Hesse potential. The last comment we need to make about the Lagrangian is that the equations of motion must be supplemented by the additional constraints

$$
q^{a} \Omega_{a b} \partial_{\mu} q^{b}=q^{a} \Omega_{a b} \partial_{\mu} \hat{q}^{b}=0
$$

and the integrability condition

$$
\partial_{\mu} \tilde{\phi}+2 \hat{q}^{a} \Omega_{a b} \partial_{\mu} \hat{q}^{b}=0
$$

The integrability condition corresponds to the requirement that the field strength of the Kaluza-Klein vector vanishes, i.e. solutions are static. For black hole solutions to the ungauged theory the constraints (2.5) correspond to a vanishing NUT charge [20].

We now perform the variation of the Lagrangian (2.1) with respect to the fields $q^{a}, \hat{q}^{a}$ and $g_{3 \mu \nu}$, and obtain the respective equations of motion

$$
\begin{aligned}
& \nabla^{\mu}\left[\tilde{H}_{a b} \partial_{\mu} q^{b}\right]-\frac{1}{2} \partial_{a} \tilde{H}_{b c}\left(\partial_{\mu} q^{b} \partial^{\mu} q^{c}-\partial_{\mu} \hat{q}^{b} \partial^{\mu} \hat{q}^{c}\right)+\partial_{a}\left(\frac{1}{4 H} V(q)\right)=0 \\
& \nabla^{\mu}\left[\tilde{H}_{a b} \partial_{\mu} \hat{q}^{b}\right]=0 \\
& \tilde{H}_{a b}\left(\partial_{\mu} q^{a} \partial_{\nu} q^{b}-\partial_{\mu} \hat{q}^{a} \partial_{\nu} \hat{q}^{b}\right)-\frac{1}{2 H} g_{\mu \nu} V(q)=-\frac{1}{2} R_{3 \mu \nu}
\end{aligned}
$$

\footnotetext{
${ }^{3}$ The exact relation is $-2 H=e^{-\mathcal{K}}$.
} 
We solve equation (2.8) immediately by setting

$$
\tilde{H}_{a b} \partial_{\mu} \hat{q}^{b}=\partial_{\mu} \mathcal{H}_{a}
$$

where $\mathcal{H}_{a}$ are harmonic functions. Using the function $\tilde{H}$ one can define a natural set of dual coordinates which simplify the remaining equations of motion. These are defined by $q_{a}:=\partial_{a} \tilde{H}$, and through the chain rule satisfy

$$
\partial_{\mu} q_{a}=\tilde{H}_{a b} \partial_{\mu} q^{b}
$$

We can relate the dual coordinates to the imaginary parts of $Y^{I}, F_{I}$ using the expressions $[20]$

$$
q_{a}=\frac{1}{H}\left(\begin{array}{c}
-v_{I} \\
u^{I}
\end{array}\right)=\frac{1}{H}\left(\begin{array}{c}
-\operatorname{Im}\left(F_{I}\right) \\
\operatorname{Im}\left(Y^{I}\right)
\end{array}\right) .
$$

The remaining equations of motion can be written in terms of the dual coordinates and harmonic functions as

$$
\begin{aligned}
\Delta q_{a}+\frac{1}{2} \partial_{a} \tilde{H}^{b c}\left(\partial_{\mu} q_{b} \partial^{\mu} q_{c}-\partial_{\mu} \mathcal{H}_{b} \partial^{\mu} \mathcal{H}_{c}\right)+\partial_{a}\left(\frac{1}{4 H} V(q)\right) & =0 \\
\tilde{H}^{a b}\left(\partial_{\mu} q_{a} \partial_{\nu} q_{b}-\partial_{\mu} \mathcal{H}_{a} \partial_{\nu} \mathcal{H}_{b}\right)-\frac{1}{2 H} g_{\mu \nu} V(q) & =-\frac{1}{2} R_{3 \mu \nu} .
\end{aligned}
$$

\subsection{Metric ansatz}

Following [5] we now make the following ansatz for the $3 \mathrm{~d}$ metric:

$$
d s_{3}^{2}=d z^{2}+e^{2 \Phi(z, w, \bar{w})} d w d \bar{w}
$$

where $\Phi$ is separable,

$$
\Phi(z, w, \bar{w})=\psi(z)+\gamma(w, \bar{w}),
$$

and $\gamma$ satisfies the Liouville equation

$$
\Delta_{(2)} \gamma \equiv 4 \partial_{w} \partial_{\bar{w}} \gamma=-\kappa e^{2 \gamma}
$$

with $\kappa$ a constant. (2.16) means that the two-metric $e^{2 \gamma} d w d \bar{w}$ has constant curvature. As a solution of (2.16) we shall take

$$
e^{2 \gamma}=\left[1+\frac{\kappa}{4} w \bar{w}\right]^{-2}
$$

We also assume that the fields $q^{a}$ and $\hat{q}^{a}$ only depend on $z$,

$$
q^{a}=q^{a}(z), \quad \hat{q}^{a}=\hat{q}^{a}(z) .
$$




\section{The $F(X)=-2 i\left(X^{0} X^{1} X^{2} X^{3}\right)^{1 / 2}$ model}

The Hesse potential for this model is given by (A.8):

$$
\begin{aligned}
H=-2\left(-\left(y_{0} x^{0}-y_{1} x^{1}-y_{2} x^{2}-y_{3} x^{3}\right)^{2}+4 y_{1} x^{1} y_{2} x^{2}+4 y_{1} x^{1} y_{3} x^{3}\right. \\
\left.+4 y_{2} x^{2} y_{3} x^{3}+4 y_{0} y_{1} y_{2} y_{3}+4 x^{0} x^{1} x^{2} x^{3}\right)^{1 / 2} .
\end{aligned}
$$

\subsection{Equations of motion}

We will consider configurations of the form

$$
x^{0}=x^{1}=x^{2}=x^{3}=0 \quad \Rightarrow \quad v_{0}=v_{1}=v_{2}=v_{3}=0 .
$$

We call such configurations axion-free since they are related to axion free configurations of the $F=-X^{1} X^{2} X^{3} / X^{0}$ model by a symplectic transformation. The matrix $\tilde{H}^{a b}$ is given by

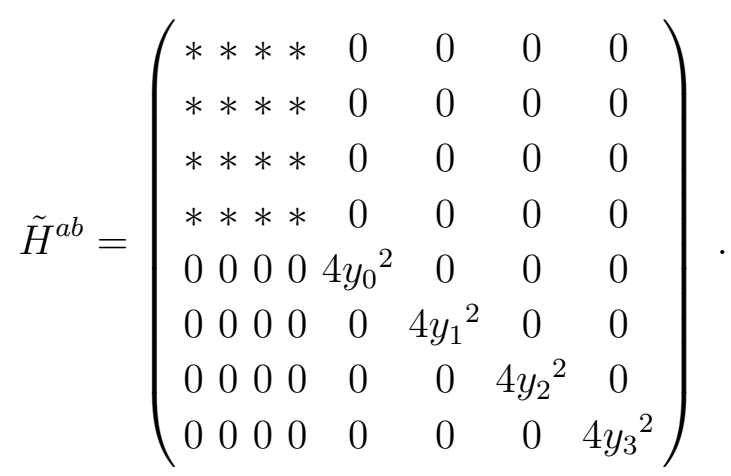

Components denoted by $*$ represent possible non-zero entries. These can easily be determined but will not play a role in the discussion, as we shall see shortly. The equations of motion greatly simplify if we impose that in addition half of the harmonic functions are constant,

$$
\partial_{\mu} \mathcal{H}_{0}=\partial_{\mu} \mathcal{H}_{1}=\partial_{\mu} \mathcal{H}_{2}=\partial_{\mu} \mathcal{H}_{3}=0
$$

From the above decomposition of $\tilde{H}^{a b}$ and $(2.10)$ we see that this condition is equivalent to switching off the electric charges. Along with the condition (3.1) this implies that the upper-left block appearing in $\tilde{H}^{a b}$ completely decouples from the equations of motion, and are therefore no longer of any relevance. The non-vanishing dual coordinates read

$$
\begin{aligned}
q_{4} & =-\frac{1}{4 y_{0}}, & q_{6} & =-\frac{1}{4 y_{2}}, \\
q_{5} & =-\frac{1}{4 y_{1}}, & q_{7} & =-\frac{1}{4 y_{3}} .
\end{aligned}
$$


The second order equations of motion (2.12) for the $q_{a}$ are given by

$$
\begin{gathered}
\Delta q_{4}-\frac{\left[\left(\partial_{z} q_{4}\right)^{2}-\left(\partial_{z} \mathcal{H}_{4}\right)^{2}\right]}{q_{4}} \\
-16 g_{0}^{2} q_{4}^{2}+16 g_{0} q_{4}^{2}\left(g_{0} q_{4}+g_{1} q_{5}+g_{2} q_{6}+g_{3} q_{7}\right)=0, \\
\Delta q_{5}-\frac{\left[\left(\partial_{z} q_{5}\right)^{2}-\left(\partial_{z} \mathcal{H}_{5}\right)^{2}\right]}{q_{5}} \\
-16 g_{1}^{2} q_{5}^{2}+16 g_{1} q_{5}^{2}\left(g_{0} q_{4}+g_{1} q_{5}+g_{2} q_{6}+g_{3} q_{7}\right)=0, \\
\Delta q_{6}-\frac{\left[\left(\partial_{z} q_{6}\right)^{2}-\left(\partial_{z} \mathcal{H}_{6}\right)^{2}\right]}{q_{6}} \\
-16 g_{2}^{2} q_{6}^{2}+16 g_{2} q_{6}^{2}\left(g_{0} q_{4}+g_{1} q_{5}+g_{2} q_{6}+g_{3} q_{7}\right)=0, \\
\Delta q_{7}-\frac{\left[\left(\partial_{z} q_{7}\right)^{2}-\left(\partial_{z} \mathcal{H}_{7}\right)^{2}\right]}{q_{7}} \\
-16 g_{3}^{2} q_{7}^{2}+16 g_{3} q_{7}^{2}\left(g_{0} q_{4}+g_{1} q_{5}+g_{2} q_{6}+g_{3} q_{7}\right)=0,
\end{gathered}
$$

and the Einstein equations (2.13) boil down to

$$
\begin{gathered}
\sum_{m=4}^{7} \frac{\left[\left(\partial_{z} q_{m}\right)^{2}-\left(\partial_{z} \mathcal{H}_{m}\right)^{2}\right]}{4 q_{m}^{2}}+4 g_{0}^{2} q_{4}^{2}+4 g_{1}^{2} q_{5}^{2}+4 g_{2}^{2} q_{6}^{2}+4 g_{3}^{2} q_{4}^{7} \\
-4\left(g_{0} q_{4}+g_{1} q_{5}+g_{2} q_{6}+g_{3} q_{7}\right)^{2}=-\partial_{z}^{2} \psi-\left(\partial_{z} \psi\right)^{2}
\end{gathered}
$$

One can check that upon making the truncation

$$
\begin{aligned}
q_{4} & \rightarrow q_{2}, & g_{0} & \rightarrow g_{0}, \\
q_{5} & \rightarrow \frac{1}{3} q_{3}, & g_{1} & \rightarrow g_{1}, \\
q_{6} & \rightarrow \frac{1}{3} q_{3}, & g_{2} & \rightarrow g_{1}, \\
q_{7} & \rightarrow \frac{1}{3} q_{3}, & g_{3} & \rightarrow g_{1},
\end{aligned}
$$

one obtains the equations of motion for the $t^{3}$ model found in [19].

Note that the fields $\mathcal{H}_{I+4}(I=0, \ldots, 3)$ are harmonic functions, i.e.

$$
\partial_{z} \mathcal{H}_{I+4}=e^{-2 \psi} C_{I+4}
$$

where the $C_{I+4}$ are constants proportional to the magnetic charges. 


\subsection{Nonextremal black holes}

In order to solve (3.4)-(3.9), we use the ansatz of [19],

$$
q_{I+4}=\frac{f_{I+4}}{e^{\psi}}, \quad f_{I+4}=z \alpha_{I+4}+\beta_{I+4},
$$

where $\alpha_{I+4}, \beta_{I+4}$ are constants, and $e^{2 \psi}$ is a quartic polynomial,

$$
e^{2 \psi}=\sum_{n=0}^{4} a_{n} z^{n}
$$

Notice that the four-dimensional geometry has two scaling symmetries, namely

$$
\left(t, z, w, e^{\phi}, e^{\psi}\right) \mapsto\left(t / \mu, \mu z, \mu w, e^{\phi} \mu^{2}, e^{\psi}\right)
$$

and

$$
\left(t, z, w, e^{\phi}, e^{\psi}\right) \mapsto\left(t / \mu, \mu z, w, e^{\phi} \mu^{2}, e^{\psi} \mu\right) .
$$

One can use the first to set $\kappa=0, \pm 1$ (corresponding to $\mathbb{R}^{2}, \mathrm{~S}^{2}$ and $\mathrm{H}^{2}$ respectively) and then the second (that leaves $\kappa$ invariant) to choose $a_{4}=1$. Furthermore, by shifting the coordinate $z$, it is always possible to eliminate the cubic term in (3.11). We shall thus take $a_{3}=0$ in what follows. After that, it is straightforward to verify that the equations of motion (3.4)-(3.9) are satisfied if the following relations for the coefficients hold:

$$
\begin{aligned}
& \alpha_{I+4}=\frac{1}{4 g_{I}}, \quad \sum_{I=0}^{3} g_{I} \beta_{I+4}=0, \quad a_{2}=\kappa-8 \sum_{I=0}^{3} g_{I}^{2} \beta_{I+4}^{2} \\
& C_{I+4}^{2}=a_{2} \beta_{I+4}^{2}+\frac{a_{0}}{16 g_{I}^{2}}-\frac{a_{1} \beta_{I+4}}{4 g_{I}}+16 g_{I}^{2} \beta_{I+4}^{4},
\end{aligned}
$$

where there is no summation over $I$ in the last equation. We are thus left with a fiveparameter family of solutions, labeled for instance by $\left(\beta_{4}, \beta_{5}, \beta_{6}, a_{0}, a_{1}\right)$. Note that the equations (2.5), (2.6) (with $\tilde{\phi}=$ const) are trivially satisfied in this case. The dilaton $\phi$ is computed from the Hesse potential,

$$
e^{\phi}=-2 H(x, y)=\frac{1}{2} e^{2 \psi}\left(f_{4} f_{5} f_{6} f_{7}\right)^{-1 / 2} .
$$

Introducing coordinates $\vartheta, \varphi$ according to

$$
w=\left\{\begin{aligned}
2 \tan \frac{\vartheta}{2} e^{i \varphi} & , \quad \kappa=1 \\
\vartheta e^{i \varphi} & , \quad \kappa=0 \\
2 \tanh \frac{\vartheta}{2} e^{i \varphi}, & \kappa=-1
\end{aligned}\right.
$$


yields for the four-dimensional metric

$$
d s_{4}^{2}=-\frac{e^{2 \psi} d t^{2}}{2\left(f_{4} f_{5} f_{6} f_{7}\right)^{1 / 2}}+2\left(f_{4} f_{5} f_{6} f_{7}\right)^{1 / 2}\left(\frac{d z^{2}}{e^{2 \psi}}+d \vartheta^{2}+S_{\kappa}^{2}(\vartheta) d \varphi^{2}\right),
$$

where we defined

$$
S_{\kappa}(\vartheta)=\left\{\begin{array}{cl}
\sin \vartheta & , \quad \kappa=1 \\
\vartheta & , \quad \kappa=0 \\
\sinh \vartheta & , \quad \kappa=-1 .
\end{array}\right.
$$

Moreover, one has from (2.2)

$$
\begin{array}{ll}
X^{0}=-\frac{i}{2 \sqrt{2}} f_{4}^{3 / 4}\left(f_{5} f_{6} f_{7}\right)^{-1 / 4}, & X^{1}=-\frac{i}{2 \sqrt{2}} f_{5}^{3 / 4}\left(f_{4} f_{6} f_{7}\right)^{-1 / 4}, \\
X^{2}=-\frac{i}{2 \sqrt{2}} f_{6}^{3 / 4}\left(f_{4} f_{5} f_{7}\right)^{-1 / 4}, & X^{3}=-\frac{i}{2 \sqrt{2}} f_{7}^{3 / 4}\left(f_{4} f_{5} f_{6}\right)^{-1 / 4} .
\end{array}
$$

Finally, from (2.3) the gauge field strengths read

$$
F_{\mu t}^{I}=G_{I \mid t w}=G_{I \mid t \bar{w}}=0, \quad G_{I \mid t z}=-\frac{C_{I+4}}{2 f_{I+4}^{2}},
$$

and using the fact that

$$
\mathcal{N}^{-1}=i\left(f_{4} f_{5} f_{6} f_{7}\right)^{-1 / 2} \operatorname{diag}\left(f_{4}^{2}, f_{5}^{2}, f_{6}^{2}, f_{7}^{2}\right),
$$

we can write this as

$$
F^{I}=\frac{i}{2} C_{I+4} e^{2 \gamma} d w \wedge d \bar{w} .
$$

Observe that the expressions for the gauge field strengths are precisely the same as for the BPS case [5]. The solution (3.14) has an event horizon at the largest root $z_{\mathrm{h}}$ of $e^{2 \psi}=0$. Regularity of the Euclidean section at $z=z_{\mathrm{h}}$ gives the Hawking temperature

$$
T=\left[\frac{\left(e^{2 \psi}\right)^{\prime}}{8 \pi\left(f_{4} f_{5} f_{6} f_{7}\right)^{1 / 2}}\right]_{z=z_{\mathrm{h}}}
$$

where a prime denotes a derivative w.r.t. $z$. Note that, if we normalize the timelike Killing vector differently in order to have the usual asymptotically AdS behaviour (cf. the mass computation below), $T$ picks a prefactor, $T \rightarrow 2 \sqrt{2}\left(g_{0} g_{1} g_{2} g_{3}\right)^{1 / 4} T$. For the Bekenstein-Hawking entropy one obtains

$$
S=\frac{A_{\mathrm{h}}}{4 G}=\frac{\left.\left(f_{4} f_{5} f_{6} f_{7}\right)^{1 / 2}\right|_{z_{\mathrm{h}}} V}{2 G},
$$


where $^{4}$

$$
V \equiv \int S_{\kappa}(\vartheta) d \vartheta d \varphi
$$

If $\kappa=1$ and the Dirac-type charge quantization condition ${ }^{5}$

$$
2 \sum_{I=0}^{3} g_{I} C_{I+4}= \pm 1
$$

holds, we can use the mass formula for so-called asymptotically magnetic AdS (mAdS) spacetimes, obtained in [27] for minimal gauged supergravity and generalized to include matter-coupling in [28]. Adapted to our conventions, it is given by $[28]^{6}$

$$
\begin{aligned}
M & =\frac{1}{8 \pi} \lim _{r \rightarrow \infty} \oint d \Sigma_{t r}\left(g^{\prime} r+\frac{g^{\prime}}{8 g^{2} r}\right)\left( \pm 2 \operatorname{Re}\left(Q_{I} X^{I}-P^{I} F_{I}\right) \sin \vartheta e_{0}^{t} e_{1}^{r} e_{2}^{\vartheta} e_{3}^{\varphi}\right. \\
& \left.+4\left|g_{I} X^{I}\right| e_{0}^{t} e_{1}^{r}+\left(\omega_{\vartheta}^{12} e_{0}^{t} e_{1}^{r} e_{2}^{\vartheta}+\omega_{\varphi}^{13} e_{0}^{t} e_{1}^{r} e_{3}^{\varphi}\right)\right) .
\end{aligned}
$$

Here we used a new radial coordinate $r=z / g^{\prime}$, with $g^{\prime}$ related to the asymptotic value of the cosmological constant by $\Lambda=-3 g^{2}=-12 \lim _{r \rightarrow \infty} g_{I} g_{J} \bar{X}^{I} X^{J}$, which yields

$$
g^{\prime}=2 \sqrt{2}\left(g_{0} g_{1} g_{2} g_{3}\right)^{1 / 4}
$$

Moreover, $t$ was rescaled, $t \rightarrow t / g^{\prime}$ in order for the metric to be asymptotically mAdS in spherical coordinates, cf. [28] for details. Note that the sign in the first line of (3.20) is the same as the one appearing in (3.19). The magnetic charges $P^{I}$ are to be identified with the constants $C_{I+4}$, whereas the electric charges $Q_{I}$ vanish in our case. Evaluating (3.20) for the solution (3.14)-(3.16), one gets

$$
\begin{aligned}
M=-\frac{1}{4 \sqrt{2}}\left(g_{0} g_{1} g_{2} g_{3}\right)^{-1 / 4}\left[a_{1} \pm 8 \sum_{I} g_{I}^{2} \beta_{I+4} C_{I+4}\right. \\
\left.\quad+96\left(g_{1} \beta_{5}+g_{2} \beta_{6}\right)\left(g_{0} \beta_{4}+g_{1} \beta_{5}\right)\left(g_{0} \beta_{4}+g_{2} \beta_{6}\right)\right]
\end{aligned}
$$

where the sign in the first line corresponds again to the one in (3.19). The mass (3.20) appears on the rhs of the anticommutator of two supercharges [28], which implies

\footnotetext{
${ }^{4}$ If the horizon is noncompact, one can still define a finite entropy density $s=S / V$.

${ }^{5}$ Note that (3.19) holds in the supersymmetric case [5], due to the minimal coupling of the gravitinos to the linear combination $g_{I} A^{I}$.

${ }^{6}$ In [28], the lower parts $F_{I}$ of the symplectic section are (asymptotically) imaginary, whereas here they are real. These two choices are related by a global $\mathrm{U}(1)$ phase rotation (which is a symmetry of the theory). Notice also that the gauge coupling constant $g$ of [28] is two times our $g$, due to an additional factor of 4 in our scalar potential w.r.t. the one in [28]. (Actually, $g$ does never appear explicitly here, since it has been absorbed into the Fayet-Iliopoulos parameters $\xi_{I}$ by $g_{I}=g \xi_{I}$, cf. [5]).
} 
that the BPS bound for asymptotically mAdS spacetimes is given by $M=0$. As a consistency check, let us verify that (3.21) vanishes in the supersymmetric case $a_{1}=0$, $a_{0}=a_{2}^{2} / 4$. (3.13), together with (3.19), leads then to

$$
C_{I+4}= \pm\left(4 g_{I} \beta_{I+4}^{2}+\frac{a_{2}}{8 g_{I}}\right) .
$$

Using this in (3.21), one obtains indeed $M=0$. However, this is not the only zero of $M$, and one might imagine for instance solutions with vanishing mass, but with $a_{1} \neq 0$, which do not correspond to any known BPS black holes in matter-coupled gauged supergravity. A similar behaviour was encountered in [29] for the $t^{3}$ model (which is included in our case), where it was argued that these solutions might be supersymmetric in extended supergravity models [30,31]. It would be interesting to see whether this is actually the case.

In [28], an alternative notion of mass was proposed, namely

$$
\begin{aligned}
M_{\mathrm{hol}} & =\frac{1}{8 \pi} \lim _{r \rightarrow \infty} \oint d \Sigma_{t r}\left(e_{[0}^{t} e_{1}^{r} e_{2]}^{\vartheta}+\sin \vartheta e_{[0}^{t} e_{1}^{r} e_{3]}^{\varphi}\right. \\
& \left.+4 g^{\prime} r\left|g_{I} X^{I}\right| e_{[0}^{t} e_{1]}^{r}+\sqrt{g^{\prime 2} r^{2}+1}\left(\omega_{\vartheta}^{a b} e_{[0}^{t} e_{a}^{r} e_{b]}^{\vartheta}+\omega_{\varphi}^{a b} e_{[0}^{t} e_{a}^{r} e_{b]}^{\varphi}\right)\right)
\end{aligned}
$$

This expression, which does not require the charge quantization condition (3.19) in order to converge, is identical to the one coming from holographic renormalization. For the black hole (3.14)-(3.16), one obtains

$$
M_{\mathrm{hol}}=-\frac{1}{4 \sqrt{2}}\left(g_{0} g_{1} g_{2} g_{3}\right)^{-1 / 4}\left[a_{1}+96\left(g_{1} \beta_{5}+g_{2} \beta_{6}\right)\left(g_{0} \beta_{4}+g_{1} \beta_{5}\right)\left(g_{0} \beta_{4}+g_{2} \beta_{6}\right)\right] \text {, }
$$

which differs from (3.21) by the term linear in the charges. The holographic renormalization procedure $[32,33]$ consists in adding boundary counterterms to the supergravity action, that lead to finite conserved charges. It would be interesting to see which finite counterterms have to be added in order to obtain the mass (3.20) instead of (3.22).

A final point we want to address in this subsection is the so-called area product formula. It was found in [21] that for a large class of rotating multi-charge black holes in asymptotically anti-de Sitter spacetimes, the product of all horizon areas (including thus also inner horizons) depends only on the charges, angular momenta and the cosmological constant. In what follows, we will show that such universal results, which may provide a 'looking glass' for probing the microscopics of general black holes [21], hold also for the solutions constructed here ${ }^{7}$. To this end, decompose $\exp (2 \psi)=\prod_{i=1}^{4}\left(z-z_{i}\right)$.

\footnotetext{
${ }^{7}$ In the case of the $t^{3}$ model, the validity of an area product formula was shown in [29].
} 
Comparison with (3.11) yields for the horizon locations $z_{i}$

$$
\begin{aligned}
& a_{0}=z_{1} z_{2} z_{3} z_{4}, \quad a_{1}=-\left(z_{1}+z_{2}\right) z_{3} z_{4}-\left(z_{3}+z_{4}\right) z_{1} z_{2}, \\
& a_{2}=z_{1} z_{2}+\left(z_{1}+z_{2}\right)\left(z_{3}+z_{4}\right)+z_{3} z_{4}, \quad z_{1}+z_{2}+z_{3}+z_{4}=0 .
\end{aligned}
$$

Using this, it is easy to see that

$$
\prod_{i=1}^{4} f_{I+4}\left(z_{i}\right)=\frac{1}{16 g_{I}^{2}}\left[\frac{a_{0}}{16 g_{I}^{2}}-\frac{a_{1} \beta_{I+4}}{4 g_{I}}+a_{2} \beta_{I+4}^{2}+16 g_{I}^{2} \beta_{I+4}^{4}\right]=\frac{C_{I+4}^{2}}{16 g_{I}^{2}},
$$

where we used (3.13) in the last step. This gives for the product of the horizon areas

$$
\prod_{i=1}^{4} A\left(z_{i}\right)=\left.\prod_{i} 2 V\left(f_{4} f_{5} f_{6} f_{7}\right)^{1 / 2}\right|_{z_{i}}=\frac{V^{4}}{16 g_{0} g_{1} g_{2} g_{3}} C_{4} C_{5} C_{6} C_{7} .
$$

Taking into account that the asymptotic value of the cosmological constant is given by $\Lambda=-3 g^{\prime 2}=-24\left(g_{0} g_{1} g_{2} g_{4}\right)^{1 / 2}$, this can be rewritten as

$$
\prod_{i=1}^{4} A\left(z_{i}\right)=\frac{36 V^{4}}{\Lambda^{2}} C_{4} C_{5} C_{6} C_{7}
$$

which depends only on the charges and the asymptotic cosmological constant. Moreover, as already noticed in [29] for the $t^{3}$ model, the dependence on the charges seems to be related to the square of the prepotential. Interestingly enough, the expression (3.25) coincides exactly with the one found in [21] for the rotating black holes in $\mathcal{N}=4 \mathrm{SO}(4)$ gauged supergravity with two pairwise equal electric charges [15], if we set the angular momentum $J$ equal to zero, replace $Q_{1}^{2} Q_{2}^{2}$ in [21] by $C_{4} C_{5} C_{6} C_{7}$ and take $V=4 \pi$ in (3.25) (spherical case $)^{8}$. This confirms the universality of the area product formula.

\subsection{First-order equations and fake superpotential}

Note that the equations of motion (3.4)-(3.7), together with (3.9), follow from an action principle with Lagrangian

$$
L=\sum_{I=0}^{3}\left[\frac{1}{4} \dot{\chi}_{I}^{2}+\frac{1}{4} C_{I+4}^{2} e^{-2 \chi_{I}}+4 g_{I}^{2} e^{2 \chi_{I}+4 \psi}\right]-\dot{\psi}^{2}-4 e^{4 \psi}\left(\sum_{I=0}^{3} g_{I} e^{\chi_{I}}\right)^{2}-\kappa e^{2 \psi} .
$$

Here, a dot denotes a derivative w.r.t. the coordinate $\zeta$ defined by $d \zeta=e^{-2 \psi} d z$, and we introduced $\chi_{I}=\ln q_{I+4}$. Eqn. (3.8) does not follow from (3.26), but the linear

\footnotetext{
${ }^{8} \Lambda$ is related to the gauge coupling constant $g$ of [21] by $\Lambda=-3 g^{2}$.
} 
combination $(3.8)+(3.9)$ is nothing else than the zero energy condition $H=0$, where $H$ is the Legendre transform of (3.26). If the potential $U$ appearing in (3.26) is given in terms of a (fake) superpotential $W$,

$$
-U=\mathcal{G}^{\alpha \beta} \frac{\partial W}{\partial \varphi^{\alpha}} \frac{\partial W}{\partial \varphi^{\beta}}
$$

where $\alpha=(I, 4), \varphi^{I}=\chi_{I}, \varphi^{4}=\psi$ and $\left(\mathcal{G}^{\alpha \beta}\right)=\operatorname{diag}(4,4,4,4,-1)$, one can rewrite the Lagrangian (up to total derivatives) in the form

$$
L=\mathcal{G}_{\alpha \beta}\left(\dot{\varphi}^{\alpha}-\mathcal{G}^{\alpha \gamma} \frac{\partial W}{\partial \varphi^{\gamma}}\right)\left(\dot{\varphi}^{\beta}-\mathcal{G}^{\beta \delta} \frac{\partial W}{\partial \varphi^{\delta}}\right),
$$

and thus the action is stationary if the first-order equations

$$
\dot{\varphi}^{\alpha}=\mathcal{G}^{\alpha \beta} \frac{\partial W}{\partial \varphi^{\beta}}
$$

hold. Notice that (3.27) is just the reduced Hamilton-Jacobi equation in the case of zero 'energy', with Hamilton's characteristic function $W^{9}$.

To solve (3.27), we make the ansatz

$$
W=\sum_{I=0}^{3}\left(a_{I} e^{-\chi_{I}}+b_{I} e^{\chi_{I}+2 \psi}\right) .
$$

Then, (3.27) is satisfied if the following conditions hold:

$$
a_{I}=\frac{1}{4} C_{I+4} \epsilon_{I}, \quad b_{I}=g_{I}, \quad \kappa=2 \sum_{I} g_{I} C_{I+4} \epsilon_{I},
$$

where $\epsilon_{I}= \pm 1$ are arbitrary signs. The last eqn. of (3.31) represents a constraint on the magnetic charges, and thus (3.30) does not solve (3.27) for arbitrary values of $C_{I+4}$. Using (3.30) and (3.31) in (3.29) gives the first-order equations

$$
\begin{aligned}
\partial_{z} q_{I+4} & =4 g_{I} q_{I+4}^{2}-\epsilon_{I} C_{I+4} e^{-2 \psi}, \quad(\text { no sum over } I) \\
\partial_{z} \psi & =-2 \sum_{I} g_{I} q_{I+4}
\end{aligned}
$$

which are solved by

$$
q_{I+4}=\frac{\alpha_{I} z+\beta_{I}}{e^{\psi}}, \quad e^{\psi}=z^{2}+c
$$

\footnotetext{
${ }^{9}$ For further discussions of the relationship between the Hamilton-Jacobi formalism and the firstorder equations derived from a fake superpotential cf. [34,35].
} 
with the constants $\alpha_{I}, \beta_{I}, c$ satisfying ${ }^{10}$

$$
\alpha_{I}=-\frac{1}{4 g_{I}}, \quad \sum_{I} g_{I} \beta_{I}=0, \quad c+16 g_{I}^{2} \beta_{I}^{2}=4 g_{I} \epsilon_{I} C_{I+4} .
$$

Summing over $I$ in the last eqn. and using (3.31) yields

$$
c=\frac{\kappa}{2}-4 \sum_{I} g_{I}^{2} \beta_{I}^{2} .
$$

We have thus a three-parameter solution (four $\beta_{I}$ 's minus the constraint $\sum_{I} g_{I} \beta_{I}=0$; (3.34) determines then $c$ and (3.33) gives the magnetic charges). Apart from these three continuous parameters, the solution is labeled also by the five discrete constants $\kappa$ and $\epsilon_{I}$. In order to have a horizon we need $c<0$ and thus

$$
8 \sum_{I} g_{I}^{2} \beta_{I}^{2}>\kappa
$$

This does always hold for $\kappa=0,-1$ (flat or hyperbolic horizon). For $\kappa=1$ the allowed values of the $\beta_{I}$ lie on the intersection of a plane through the origin (second eqn. of (3.33) with the exterior of a ball in $\mathbb{R}^{4}$, eqn. (3.35). Since $e^{2 \psi}$ has a double root, the resulting black hole is extremal. If all signs $\epsilon_{I}$ are equal, it reduces to the supersymmetric solution found in [5].

\subsection{Recovering the four-charge solution of $\mathcal{N}=8$ gauged supergravity}

Let us now show how the nonextremal black holes of section 3.2 contain the magnetic four-charge solution to $\mathcal{N}=8$ gauged supergravity presented in [14]. After a $U(1)^{4}$ truncation, this theory boils down to the $\mathcal{N}=2$ model considered here, with prepotential $F=-2 i\left(X^{0} X^{1} X^{2} X^{3}\right)^{1 / 2}$, and all $g_{I}$ equal. The metric, moduli and gauge fields read (cf. eqn. (6.2) of [14])

$$
\begin{aligned}
& d s^{2}=-\left(H_{0} H_{1} H_{2} H_{3}\right)^{-1 / 2} f d t^{2}+\left(H_{0} H_{1} H_{2} H_{3}\right)^{1 / 2}\left(\frac{d r^{2}}{f}+r^{2} d \Omega^{2}\right) \\
& e^{2 \phi^{(12)}}=\frac{H_{2} H_{3}}{H_{0} H_{1}}, \quad e^{2 \phi^{(13)}}=\frac{H_{1} H_{3}}{H_{0} H_{2}}, \quad e^{2 \phi^{(14)}}=\frac{H_{1} H_{2}}{H_{0} H_{3}}, \\
& H_{I}=1+\frac{k \sinh ^{2} \mu_{I}}{r}, \quad f=1-\frac{k}{r}+2 g^{2} r^{2} H_{0} H_{1} H_{2} H_{3} \\
& F_{\vartheta \varphi}^{I}=\frac{\eta_{I}}{2 \sqrt{2}} k \cosh \mu_{I} \sinh \mu_{I} \sin \vartheta .
\end{aligned}
$$

\footnotetext{
${ }^{10}$ Here, $\alpha_{I}$ is negative while $\alpha_{I+4}$ in $(3.10)$ is positive. $\alpha_{I}>0$ can easily be achieved by taking $z \rightarrow-z$.
} 
Here, $\eta_{I}= \pm 1$ are arbitrary signs, the $\mu_{I}$ determine the magnetic charges, and $k$ is a kind of nonextremality parameter. After the identification $g=2 g_{I}$ and the coordinate transformation

$$
r=\frac{z}{\sqrt{2} g}-\frac{k}{4} \sum_{I=0}^{3} \sinh ^{2} \mu_{I},
$$

the solution (3.36) takes the form of the one in section 3.2, with

$$
\begin{aligned}
\beta_{I+4}= & \frac{k}{\sqrt{2}}\left(\sinh ^{2} \mu_{I}-\frac{1}{4} \sum_{J} \sinh ^{2} \mu_{J}\right) \\
a_{0}= & \frac{g^{2} k^{2}}{2}\left[\left(\frac{1}{2} \sum_{I} \sinh ^{2} \mu_{I}\right)^{2}+\sum_{I} \sinh ^{2} \mu_{I}\right]+16 g^{4} \beta_{4} \beta_{5} \beta_{6} \beta_{7} \\
a_{1}= & -\sqrt{2} g k\left(1+\frac{1}{2} \sum_{I} \sinh ^{2} \mu_{I}\right)-\sqrt{2} \frac{g^{3} k^{3}}{4}\left(\sinh ^{2} \mu_{0}+\sinh ^{2} \mu_{1}-\sinh ^{2} \mu_{2}\right. \\
& \left.-\sinh ^{2} \mu_{3}\right)\left[\left(\sinh ^{2} \mu_{2}-\sinh ^{2} \mu_{3}\right)^{2}-\left(\sinh ^{2} \mu_{0}-\sinh ^{2} \mu_{1}\right)^{2}\right] \\
a_{2}= & 1-2 g^{2} \sum_{I} \beta_{I+4}^{2} \cdot
\end{aligned}
$$

Since both (3.36) and the black holes obtained in 3.2 are labeled by five continuous parameters $\left(k, \mu_{I}\right.$ for $\left.(3.36)\right)$, one might wonder if they are not equivalent (if all $g_{I}$ are equal; for generic coupling constants the solution (3.14)-(3.16) is clearly more general). This is however not the case: Suppose for instance that all charges are equal in (3.36). Then, the 'harmonic' functions $H_{I}$ coincide as well, and thus the scalar fields are constant. In the solution of section 3.2 instead, one can have equal charges and yet nontrivial profiles for the moduli (take e.g. $a_{1}=0, \beta_{4}=\beta_{5}=-\beta_{6}=-\beta_{7}$ ). Moreover, (3.14)-(3.16) contains a subclass of black holes that are BPS, while it was shown in [14] that (3.36) can never be supersymmetric. To understand better what happens, let us consider the subcase $\beta_{6}=\beta_{4}, \beta_{7}=\beta_{5}, \mu_{2}=\mu_{0}, \mu_{3}=\mu_{1}$, that implies $X^{2}=X^{0}$, $X^{3}=X^{1}, C_{6}=C_{4}, C_{7}=C_{5}$, leading to the $F=-i X^{0} X^{1}$ model considered in [19]. Since all $g_{I}$ are equal $\left(2 g_{I}=g\right)$, the second equ. of (3.12) boils down to $\sum_{I} \beta_{I+4}=0$ and thus $\beta_{5}=-\beta_{4}$. From (3.13) one obtains then

$$
C_{5}^{2}-C_{4}^{2}=\frac{a_{1}}{g} \beta_{4}
$$

If the charges are equal (up to a sign), $C_{5}^{2}=C_{4}^{2}$, and we have therefore $a_{1}=0$ or $\beta_{4}=0$. The former case is (for $C_{5}=C_{4}$ ) the supersymmetric black hole found in [5] (with running scalar), whereas the latter corresponds to the Duff-Liu solution (3.36), 
with constant scalar profiles. In this context, notice also that in the parametrization (3.37), for $\mu_{2}=\mu_{0}, \mu_{3}=\mu_{1}$, we get

$$
a_{1}=-\sqrt{2} g k\left(1+\sinh ^{2} \mu_{0}+\sinh ^{2} \mu_{1}\right)
$$

which is always nonvanishing (if $k \neq 0$ ), and thus the supersymmetric case cannot appear.

In conclusion, the solution (3.14)-(3.16) includes both (3.36) and the BPS black holes constructed in [5].

\section{The $F(X)=-X^{1} X^{2} X^{3} / X^{0}$ model}

In [20] the Hesse potential for the $F(X)=-X^{1} X^{2} X^{3} / X^{0}$ model was calculated to be

$$
\begin{aligned}
H=-2\left(-\left(y_{0} x^{0}+y_{1} x^{1}+y_{2} x^{2}\right.\right. & \left.+y_{3} x^{3}\right)^{2}+4 y_{1} x^{1} y_{2} x^{2}+4 y_{1} x^{1} y_{3} x^{3} \\
& \left.+4 y_{2} x^{2} y_{3} x^{3}+4 x^{0} y_{1} y_{2} y_{3}-4 y_{0} x^{1} x^{2} x^{3}\right)^{1 / 2} .
\end{aligned}
$$

\subsection{Equations of motion}

We will consider solutions which take the form

$$
y_{0}=x^{1}=x^{2}=x^{3}=0 \quad \Rightarrow \quad u^{0}=v_{1}=v_{2}=v_{3}=0
$$

This ensures that the kinetic term in the Lagranian does not transform under any axion-like shift symetries of the physical scalar fields. The matrix $\tilde{H}^{a b}$ is given by

$$
\tilde{H}^{a b}=\left(\begin{array}{cccccccc}
4 x^{0^{2}} & 0 & 0 & 0 & 0 & 0 & 0 & 0 \\
0 & * & * & * & * & 0 & 0 & 0 \\
0 & * & * & * & * & 0 & 0 & 0 \\
0 & * & * & * & * & 0 & 0 & 0 \\
0 & * & * & * & * & 0 & 0 & 0 \\
0 & 0 & 0 & 0 & 0 & 4 y_{1}^{2} & 0 & 0 \\
0 & 0 & 0 & 0 & 0 & 0 & 4 y_{2}^{2} & 0 \\
0 & 0 & 0 & 0 & 0 & 0 & 0 & 4 y_{3}^{2}
\end{array}\right) .
$$

The equations of motion greatly simplify if we impose that in addition half of the harmonic functions are constant,

$$
\partial_{\mu} \mathcal{H}_{1}=\partial_{\mu} \mathcal{H}_{2}=\partial_{\mu} \mathcal{H}_{3}=\partial_{\mu} \mathcal{H}_{4}=0
$$


Using the decomposition of $\tilde{H}^{a b}$ and $(2.10)$ one can see that this condition is equivalent to switching off the electric charges $Q_{1}, Q_{2}, Q_{3}$ and the magentic charge $P^{1}$. Moreover, it implies that block in $\tilde{H}^{a b}$ consisting of entries denoted by $*$ completely decouples from the equations of motion. The non-vanishing dual coordinates read

$$
\begin{aligned}
q_{0} & =-\frac{1}{4 x^{0}}, & q_{6} & =-\frac{1}{4 y_{2}}, \\
q_{5} & =-\frac{1}{4 y_{1}}, & q_{7} & =-\frac{1}{4 y_{3}} .
\end{aligned}
$$

The second order equations of motion (2.12) for the $q_{a}$ are given by

$$
\begin{gathered}
\Delta q_{0}-\frac{\left[\left(\partial_{z} q_{0}\right)^{2}-\left(\partial_{z} \mathcal{H}_{0}\right)^{2}\right]}{q_{0}}=0, \\
\Delta q_{5}-\frac{\left[\left(\partial_{z} q_{5}\right)^{2}-\left(\partial_{z} \mathcal{H}_{5}\right)^{2}\right]}{q_{5}} \\
+16 g_{1} q_{5}^{2}\left(g_{2} q_{6}+g_{3} q_{7}\right)=0, \\
\Delta q_{6}-\frac{\left[\left(\partial_{z} q_{6}\right)^{2}-\left(\partial_{z} \mathcal{H}_{6}\right)^{2}\right]}{q_{6}} \\
+16 g_{2} q_{6}^{2}\left(g_{1} q_{5}+g_{3} q_{7}\right)=0, \\
\Delta q_{7}-\frac{\left[\left(\partial_{z} q_{7}\right)^{2}-\left(\partial_{z} \mathcal{H}_{7}\right)^{2}\right]}{q_{7}} \\
+16 g_{3} q_{7}^{2}\left(g_{1} q_{5}+g_{2} q_{6}\right)=0,
\end{gathered}
$$

and the Einstein equations (2.13) become

$$
\begin{aligned}
& \frac{\left[\left(\partial_{z} q_{0}\right)^{2}-\left(\partial_{z} \mathcal{H}_{0}\right)^{2}\right]}{4 q_{0}^{2}}+\frac{\left[\left(\partial_{z} q_{5}\right)^{2}-\left(\partial_{z} \mathcal{H}_{5}\right)^{2}\right]}{4 q_{5}^{2}} \\
& +\frac{\left[\left(\partial_{z} q_{6}\right)^{2}-\left(\partial_{z} \mathcal{H}_{6}\right)^{2}\right]}{4 q_{6}^{2}}+\frac{\left[\left(\partial_{z} q_{7}\right)^{2}-\left(\partial_{z} \mathcal{H}_{7}\right)^{2}\right]}{4 q_{7}^{2}} \\
& \quad-8 g_{1} g_{2} q_{5} q_{6}-8 g_{1} g_{3} q_{5} q_{7}-8 g_{2} g_{3} q_{6} q_{7}=-\partial_{z}^{2} \psi-\left(\partial_{z} \psi\right)^{2}, \\
& 16 g_{1} g_{2} q_{5} q_{6}+16 g_{1} g_{3} q_{5} q_{7}+16 g_{2} g_{3} q_{6} q_{7}=\partial_{z}^{2} \psi+2\left(\partial_{z} \psi\right)^{2}-\kappa e^{-2 \psi} .
\end{aligned}
$$

One can check that upon making the truncation

$$
\begin{array}{ll}
q_{0} \rightarrow q_{0}, & g_{0} \rightarrow g_{0}, \\
q_{5} \rightarrow \frac{1}{3} q_{3}, & g_{1} \rightarrow g_{1}, \\
q_{6} \rightarrow \frac{1}{3} q_{3}, & g_{2} \rightarrow g_{1}, \\
q_{7} \rightarrow \frac{1}{3} q_{3}, & g_{3} \rightarrow g_{1},
\end{array}
$$


one obtains the equations of motion for the $F=-X^{1^{3}} / X^{0}$ model found in [19].

Note that the fields $\mathcal{H}_{0}, \mathcal{H}_{5}, \mathcal{H}_{6}, \mathcal{H}_{7}$ are harmonic functions, i.e.

$$
\partial_{z} \mathcal{H}_{0}=-D e^{-2 \psi}, \quad \partial_{z} \mathcal{H}_{5}=C_{5} e^{-2 \psi}, \quad \partial_{z} \mathcal{H}_{6}=C_{6} e^{-2 \psi}, \quad \partial_{z} \mathcal{H}_{7}=C_{7} e^{-2 \psi} .
$$

where the $C_{5,6,7}$ are constants proportional to the magnetic charges and $D$ is a constant proportional to the electric charge.

\subsection{First-order equations and fake superpotential}

As was explained in [19], (4.4) is nothing else than the Liouville equation, with the solution

$$
q_{0}=D \frac{\sin p \zeta}{p}
$$

where again $d \zeta=e^{-2 \psi} d z$, and $p$ (with $p^{2}$ real) is the Liouville momentum. Using this, the Einstein equation (4.8) boils down to

$$
\begin{gathered}
\frac{p^{2}}{4} e^{-4 \psi}+\sum_{\alpha=5}^{7} \frac{\left(\partial_{z} q_{\alpha}\right)^{2}-C_{\alpha}^{2} e^{-4 \psi}}{4 q_{\alpha}^{2}}-8 g_{1} g_{2} q_{5} q_{6}-8 g_{1} g_{3} q_{5} q_{7}-8 g_{2} g_{3} q_{6} q_{7} \\
=-\partial_{z}^{2} \psi-\left(\partial_{z} \psi\right)^{2}
\end{gathered}
$$

and thus $q_{0}$ decouples completely from the other fields.

The equations of motion (4.5)-(4.7), together with (4.9), follow from an action principle with the Toda-like Lagrangian

$$
\begin{aligned}
L & =-\dot{\psi}^{2}+\frac{1}{4} \sum_{\alpha=5}^{7}\left(\left(\dot{\chi}^{\alpha}\right)^{2}+C_{\alpha}^{2} e^{-2 \chi^{\alpha}}\right) \\
& -8 e^{4 \psi}\left(g_{1} g_{2} e^{\chi^{5}+\chi^{6}}+g_{1} g_{3} e^{\chi^{5}+\chi^{7}}+g_{2} g_{3} e^{\chi^{6}+\chi^{7}}\right)-\kappa e^{2 \psi},
\end{aligned}
$$

where a dot denotes a derivative w.r.t. $\zeta$, and we defined $\chi^{\alpha} \equiv \ln q_{\alpha}$. Notice that (4.11) does not follow from (4.12), but the linear combination (4.11)+(4.9) is exactly the 'energy conservation' $H=-p^{2} / 4 \equiv E$, with $H$ the Legendre transform of $L$. It is amusing to note that the 'energy' is essentially the square of the Liouville momentum.

Defining the metric $\mathcal{G}$ by $\left(\mathcal{G}^{\alpha \beta}\right)=\operatorname{diag}(4,4,4), \mathcal{G}^{\psi \psi}=-1, \mathcal{G}^{\alpha \psi}=0$, one can write

$$
L=\mathcal{G}_{\alpha \beta} \dot{\chi}^{\alpha} \dot{\chi}^{\beta}+\mathcal{G}_{\psi \psi} \dot{\psi}^{2}-U
$$

with $U$ the potential term appearing in (4.12). Then, the reduced Hamilton-Jacobi equation reads

$$
\mathcal{G}^{\alpha \beta} \frac{\partial W}{\partial \chi^{\alpha}} \frac{\partial W}{\partial \chi^{\beta}}+\mathcal{G}^{\psi \psi}\left(\frac{\partial W}{\partial \psi}\right)^{2}+U=E,
$$


and we have the first-order equations

$$
\dot{\chi}^{\alpha}=\mathcal{G}^{\alpha \beta} \frac{\partial W}{\partial \chi^{\beta}}, \quad \dot{\psi}=\mathcal{G}^{\psi \psi} \frac{\partial W}{\partial \psi} .
$$

If $E=0$ (i.e., $p=0$ ) and the generalized 'charge quantization condition'

$$
\sum_{\alpha=5}^{7} g_{\alpha-4} \epsilon_{\alpha} C_{\alpha}=\frac{\kappa}{2}
$$

holds, where $\epsilon_{\alpha}= \pm 1$ are arbitrary signs, a particular solution to (4.14) is given by

$$
W=\sum_{\alpha=5}^{7}\left(\frac{\epsilon_{\alpha} C_{\alpha}}{4} e^{-\chi^{\alpha}}+g_{\alpha-4} e^{\chi^{\alpha}+2 \psi}\right)
$$

which has exactly the same form as the fake superpotential (3.30) for the model considered in section 3, the only (but crucial) difference being that the sum in (3.30) involves four terms, whereas in (4.17) one has only three ${ }^{11}$. There seems thus to be a universal structure behind the form of the fake superpotential. We shall come back to this point later.

With (4.17), the first-order equations (4.15) become

$$
\partial_{z} \psi=-2 \sum_{\alpha} g_{\alpha-4} q_{\alpha}, \quad \partial_{z} q_{\alpha}=4 g_{\alpha-4} q_{\alpha}^{2}-\epsilon_{\alpha} C_{\alpha} e^{-2 \psi}
$$

which differ from the BPS equations (3.69) and (3.70) of [5] only by the signs $\epsilon_{\alpha}{ }^{12}$. For $\epsilon_{\alpha}$ all equal one recovers the BPS case.

A special solution of $(4.18)$ is

$$
e^{\psi}=z, \quad q_{\alpha}=\frac{\pi_{\alpha}}{z},
$$

where the constants $\pi_{\alpha}$ satisfy

$$
4 g_{\alpha-4} \pi_{\alpha}^{2}+\pi_{\alpha}-\epsilon_{\alpha} C_{\alpha}=0
$$

Note that the equations (2.5), (2.6) (with $\tilde{\phi}=0$ ) are trivially satisfied in this case. The dilaton $\phi$ is computed from the Hesse potential,

$$
e^{\phi}=-2 H(x, y)=\frac{1}{2}\left(q_{0} q_{5} q_{6} q_{7}\right)^{-1 / 2} .
$$

\footnotetext{
${ }^{11}$ We shall see below that this affects the asymptotics of the resulting black hole.

${ }^{12}$ The dictionary to get from (4.18) (with $\epsilon_{\alpha}=-1$ ) to (3.69) and (3.70) of [5] is $q_{\alpha}=2 i H^{\alpha-4}$, $C_{\alpha}=4 \pi p^{\alpha-4}$.
} 
This yields for the four-dimensional metric

$$
d s_{4}^{2}=-\frac{z^{3 / 2} d t^{2}}{2\left(q_{0} \pi_{5} \pi_{6} \pi_{7}\right)^{1 / 2}}+\frac{2\left(q_{0} \pi_{5} \pi_{6} \pi_{7}\right)^{1 / 2}}{z^{3 / 2}}\left(d z^{2}+z^{2}\left(d \vartheta^{2}+S_{\kappa}^{2}(\vartheta) d \varphi^{2}\right)\right) .
$$

Here, $q_{0}$ is determined by (4.10) (in the limit $p \rightarrow 0$ ), which gives

$$
q_{0}=h_{0}-\frac{D}{z}
$$

where $h_{0}$ is an integration constant, and the coordinates $\vartheta, \varphi$ as well as the function $S_{\kappa}(\vartheta)$ were defined in section 3.2 .

The solution (4.21) has an event horizon at $z=0$. The near-horizon geometry is $\mathrm{AdS}_{2} \times \Sigma$, where $\Sigma$ is a two-space of constant curvature. Note that, for $z \rightarrow \infty$, the spacetime does not approach AdS. In the special case $\epsilon_{\alpha}=-1, g_{1} C_{5}=g_{2} C_{6}=g_{3} C_{7}$, $g_{1} q_{5}=g_{2} q_{6}=g_{3} q_{7}$ one recovers the BPS black hole (3.81) of [5] with $C=0$.

The scalar fields follow from (2.2), with the result

$$
X^{0}=-\frac{\left(q_{0} q_{5} q_{6} q_{7}\right)^{1 / 4}}{2 \sqrt{2} q_{0}}, \quad X^{\alpha-4}=-\frac{i q_{\alpha}}{2 \sqrt{2}\left(q_{0} q_{5} q_{6} q_{7}\right)^{1 / 4}} .
$$

Finally, from (2.3) the nonvanishing components of the gauge field strengths read

$$
F_{t z}^{0}=-\frac{1}{2} \partial_{z}\left(q_{0}^{-1}\right), \quad G_{\alpha-4 \mid t z}=-\frac{C_{\alpha}}{2 \pi_{\alpha}^{2}},
$$

and using the fact that

$$
\mathcal{N}^{-1}=i\left(q_{0} q_{5} q_{6} q_{7}\right)^{-1 / 2} \operatorname{diag}\left(q_{0}^{-1} q_{5} q_{6} q_{7}, q_{5}^{2}, q_{6}^{2}, q_{7}^{2}\right)
$$

we can write this as

$$
F^{0}=-\frac{1}{2} d t \wedge d\left(q_{0}^{-1}\right), \quad F^{\alpha-4}=\frac{i}{2} C_{\alpha} e^{2 \gamma} d w \wedge d \bar{w} .
$$

Observe that the expressions for the gauge field strengths are precisely the same as for the BPS case [5]. Since the black hole (4.21) is extremal, its temperature is zero. For the Bekenstein-Hawking entropy one obtains

$$
S=\frac{A_{\mathrm{h}}}{4 G}=\frac{\left(-D \pi_{5} \pi_{6} \pi_{7}\right)^{1 / 2} V}{2 G},
$$

where $V$ was defined in (3.18). 


\section{Fake superpotential for arbitrary prepotential}

For the three-dimensional base space metric given in section 2.2, the equations of motion (2.12), (2.13) reduce to

$$
\begin{aligned}
& e^{-2 \psi} \partial_{z}\left(e^{2 \psi} \partial_{z} q_{a}\right)+\frac{1}{2} \partial_{a} \tilde{H}^{b c}\left(\partial_{z} q_{b} \partial_{z} q_{c}-C_{a} C_{b} e^{-4 \psi}\right)+\partial_{a}\left(\frac{V(q)}{4 H}\right)=0, \\
& \tilde{H}^{a b}\left(\partial_{z} q_{a} \partial_{z} q_{b}-C_{a} C_{b} e^{-4 \psi}\right)-\frac{1}{2 H} V(q)=-\partial_{z}^{2} \psi-\left(\partial_{z} \psi\right)^{2}, \\
& \frac{1}{H} V(q)=\partial_{z}^{2} \psi+2\left(\partial_{z} \psi\right)^{2}-\kappa e^{-2 \psi},
\end{aligned}
$$

where we took into account that the $\mathcal{H}_{a}$ are harmonic functions on the base, i.e., $\partial_{z} \mathcal{H}_{a}=C_{a} e^{-2 \psi}$, with charges $C_{a}$. Eqns. (5.1) and (5.3) follow from an action principle with Lagrangian

$$
L=\tilde{H}^{a b} \dot{q}_{a} \dot{q}_{b}-\dot{\psi}^{2}+\tilde{H}^{a b} C_{a} C_{b}-\frac{V}{2 H} e^{4 \psi}-\kappa e^{2 \psi}
$$

where, as before, a dot denotes a derivative w.r.t. the coordinate $\zeta$ defined by $d \zeta=$ $e^{-2 \psi} d z$. Notice that (5.2) does not follow from (5.4), but the linear combination $(5.2)+(5.3)$ is precisely the 'zero-energy condition' $H=0$, with $H$ the Legendre transform of $L$. Let us define the index $\alpha=(a, 2 n+2)$ (i.e., $\alpha=0, \ldots, 2 n+2)$, the fields $\varphi_{\alpha}$ with $\varphi_{a}=q_{a}, \varphi_{2 n+2}=\psi$, and the 'metric'

$$
\left(\mathcal{G}_{\alpha \beta}\right)=\left(\begin{array}{cc}
\tilde{H}_{a b} & 0 \\
0 & -1
\end{array}\right) .
$$

If the potential $U$ appearing in (5.4) is given in terms of a fake superpotential $W$,

$$
-U=\mathcal{G}_{\alpha \beta} \frac{\partial W}{\partial \varphi_{\alpha}} \frac{\partial W}{\partial \varphi_{\beta}}
$$

one can rewrite the Lagrangian $L$ (up to total derivatives) in the form

$$
L=\mathcal{G}^{\alpha \beta}\left(\dot{\varphi}_{\alpha}-\mathcal{G}_{\alpha \gamma} \frac{\partial W}{\partial \varphi_{\gamma}}\right)\left(\dot{\varphi}_{\beta}-\mathcal{G}_{\beta \delta} \frac{\partial W}{\partial \varphi_{\delta}}\right),
$$

and thus the action is stationary if the first-order equations

$$
\dot{\varphi}_{\alpha}=\mathcal{G}_{\alpha \gamma} \frac{\partial W}{\partial \varphi_{\gamma}}
$$

hold. Again, (5.5) is just the reduced Hamilton-Jacobi equation in the case of zero 'energy', with Hamilton's characteristic function $W$. Using the expression (2.4) for the 
Fayet-Iliopoulos potential in terms of the Hesse potential, and imposing the zero-axion condition $x^{I}=0$, it is straightforward to show that a particular solution to (5.5) is given by

$$
W=q^{a} S_{a}^{b} C_{b}+k^{a} q_{a} e^{2 \psi},
$$

where $k^{a}=\left(0, \ldots, 0, \pm g_{I}\right)^{T}$, and $S_{a}{ }^{b}$ denotes a constant 'field rotation matrix' that must satisfy the compatibility condition

$$
\tilde{H}^{c d}=\tilde{H}^{a b} S_{a}{ }^{c} S_{b}{ }^{d}
$$

with $\tilde{H}^{a b}$. Moreover, in order for (5.8) to satisfy (5.5), the Dirac-type charge quantization condition

$$
2 k^{a} S_{a}^{b} C_{b}=-\kappa
$$

must hold. A nontrivial $S$ (different from \pm Id) allows to generate new solutions from known ones by 'rotating charges'. This technique was first introduced in [36,37], and generalizes the sign-flipping procedure of [38], which was used in the preceding two sections. Note that in general it is not guaranteed that a nontrivial field rotation matrix satisfying (5.9) exists. Geometrically, this is equivalent to the problem of identifying totally geodesic, totally isotropic submanifolds of the scalar target space [20]. For instance, it was established in [20] that a nontrivial solution to (5.9) always exists for non-axionic solutions of models with a prepotential of the form $F=f\left(Y^{1}, \ldots, Y^{n}\right) / Y^{0}$, where $f$ is real when evaluated on real fields. Below we will show that $S= \pm$ Id leads precisely to the BPS solutions of [5].

Before we proceed, a short comment on the ungauged limit is in order. In this case, $\kappa=k^{a}=0$ and thus (5.10) holds identically. The (fake) superpotential reduces to $W=q^{a} S_{a}{ }^{b} C_{b}$. If we restrict to BPS solutions, we can take without loss of generality $S_{a}{ }^{b}=\delta_{a}{ }^{b}$ (the other sign corresponds just to a redefiniton $z \rightarrow-z$ ). Since $\psi=0$, the Hamilton-Jacobi equations boil down to $\partial_{z} q_{a}=\tilde{H}_{a b} \partial_{q_{b}} W=C_{a}$. On-shell one has therefore $W=q^{a} \partial_{z} q_{a}$. Taking into account that $q^{a} q_{a}=-1$ and $q_{a}=\partial_{a} \tilde{H}$, we get thus $W=-\partial_{z} \tilde{H}$, so Hamilton's characteristic function is just minus the derivative of the Hesse potential.

Coming back to the gauged case, the flow equations (5.7) read

$$
\begin{gathered}
\dot{q}_{a}=S_{a}{ }^{b} C_{b}+\tilde{H}_{a b} k^{b} e^{2 \psi}, \\
\dot{\psi}=-2 k^{a} q_{a} e^{2 \psi}=\mp 2 g_{I} \frac{\partial \tilde{H}}{\partial y_{I}} e^{2 \psi} .
\end{gathered}
$$

Of course, these have to be supplemented by the constraints (2.5) and the integrability condition (2.6). While the first equ. of (2.5) holds automatically provided $x^{I}=0$, the 
second equ. of (2.5) and (2.6) become respectively

$$
q^{a} \Omega_{a b} \tilde{H}^{b c} C_{c}=0, \quad \hat{q}^{a} \Omega_{a b} \tilde{H}^{b c} C_{c}=0 .
$$

Using $\psi$ in place of $z$ as a radial coordinate, we can finally write for the metric ${ }^{13}$

$$
d s_{4}^{2}=2 H d t^{2}-\frac{1}{2 H}\left[\left(2 g_{I} \frac{\partial \tilde{H}}{\partial y_{I}}\right)^{-2} d \psi^{2}+e^{2 \psi}\left(d \vartheta^{2}+S_{\kappa}^{2}(\vartheta) d \varphi^{2}\right)\right],
$$

while the scalars satisfy the flow equation

$$
\frac{d q_{a}}{d \psi}=-\frac{1}{2 k^{c} q_{c}}\left(S_{a}^{b} C_{b} e^{-2 \psi}+\tilde{H}_{a b} k^{b}\right) .
$$

In order to specify the full solution, one also has to give the expression for the fluxes, which we did not write down here.

Let us now show that for $S= \pm \mathrm{Id}$, one recovers the supersymmetric black holes constructed in [5]. First of all, it is easy to see that (5.12) corresponds exactly to equ. (2.22) of [5] if we take the upper sign and identify the field $b$ used in [5] with $e^{\phi / 2}$. Moreover, taking into account that

$$
\tilde{H}_{a b}=\frac{1}{H} \hat{H}_{a b}-\frac{2}{H^{2}} \Omega_{a c} q^{c} \Omega_{b d} q^{d},
$$

as well as

$$
\hat{H}_{a b}=\left(\begin{array}{cc}
\operatorname{Im} \mathcal{N}+\operatorname{Re} \mathcal{N}(\operatorname{Im} \mathcal{N})^{-1} \operatorname{Re} \mathcal{N} & -\operatorname{Re} \mathcal{N}(\operatorname{Im} \mathcal{N})^{-1} \\
-(\operatorname{Im} \mathcal{N})^{-1} \operatorname{Re} \mathcal{N} & (\operatorname{Im} \mathcal{N})^{-1}
\end{array}\right),
$$

together with $k^{a} \Omega_{a b} q^{b}=0$ for non-axionic solutions and $C_{a} \equiv 4 \pi\left(q_{I},-p^{I}\right)^{T}$, one may check that (5.11) is equivalent to eqns. (2.20) and (2.21) of [5] for $S_{a}{ }^{b}=-\delta_{a}{ }^{b}$. Finally, the staticity condition (2.19) of [5] can be rewritten as $\dot{q}_{a} \Omega^{a b} q_{b}=0$, which is also satisfied by virtue of (5.11), $C_{a} \Omega^{a b} q_{b}=0$ and $k^{a} \tilde{H}_{a b} \Omega^{b c} q_{c}=0$. Note that the last two equations can be shown by using $H_{a b} \Omega^{b c} H_{c d}=-4 \Omega_{a d}$,

$$
\tilde{H}_{a b}=-\frac{1}{2 H} H_{a b}+\frac{1}{2 H^{2}} H_{a} H_{b},
$$

as well as the first relation of (5.13).

We now wish to compare the fake superpotential (5.8) with results that appeared previously in the literature. The authors of $[6]^{14}$ rewrote the supergravity action (reduced to one dimension) as a sum of squares of first-order differential conditions. This

\footnotetext{
${ }^{13}$ Note that $H<0$.

${ }^{14}$ See also [39] for related work in the case of brane solutions $(\kappa=0)$. In five-dimensional gauged supergravity, the formalism of first-order flow equations was used in [40] and more recently for extremal black branes in [41].
} 
was done for a generic prepotential, but was possible only provided a certain supersymmetry constraint ((2.27) of [6], corresponds essentially to (5.10) with $S= \pm$ Id) holds. The resulting first-order equations involve the 'superpotential'15

$$
W=e^{\phi / 2}\left|\mathcal{Z}-i e^{2 \psi-\phi} \mathcal{L}\right|
$$

where $\mathcal{Z}=Q_{I} X^{I}-P^{I} F_{I}$ denotes the central charge, $Q_{I}$ and $P^{I}$ are the electric and magnetic charges respectively, and $\mathcal{L}=2 X^{I} g_{I}$ is the actual superpotential defining the scalar potential according to

$$
V=g^{\alpha \bar{\beta}} \mathcal{D}_{\alpha} \mathcal{L} \mathcal{D}_{\bar{\beta}} \overline{\mathcal{L}}-3|\mathcal{L}|^{2}
$$

Now, using $C_{a}=\left(Q_{I},-P^{I}\right)^{T}$, one shows that the central charge obeys

$$
e^{\phi / 2} \mathcal{Z}=\left(q^{a}-i H \Omega^{a b} q_{b}\right) C_{a},
$$

which in our case boils down to $e^{\phi / 2} \mathcal{Z}=q^{a} C_{a}$ since (as already pointed out above) $C_{a} \Omega^{a b} q_{b}$ vanishes here. Furthermore, for axion-free solutions one has $e^{\phi / 2} g_{I} X^{I}=$ $\mp i e^{\phi} k^{a} q_{a} / 2$, and thus

$$
e^{\phi / 2}\left(\mathcal{Z}-i e^{2 \psi-\phi} \mathcal{L}\right)=q^{a} C_{a} \mp k^{a} q_{a} e^{2 \psi}
$$

This coincides with (5.8) if we take the lower sign and $S_{a}{ }^{b}=\delta_{a}{ }^{b}$, or for the upper sign and $S_{a}{ }^{b}=-\delta_{a}{ }^{b}$ (plus an inversion $z \rightarrow-z$ ). In conclusion, we saw that our fake superpotential (5.8), which is identical to Hamilton's characteristic function in a Hamilton-Jacobi formalism, matches in the supersymmetric case (where $S= \pm \mathrm{Id}$ ) the superpotential (5.16) proposed in [6].

For the $F=-X^{1} X^{2} X^{3} / X^{0}$ model considered in section 4 , things are more subtle since we kept $x^{0} \neq 0$ such that the zero-axion condition is not satisfied. The analysis above does therefore not apply. With only $Q_{0}, P^{1,2,3}$ nonvanishing, and the truncation $F_{0}, X^{1,2,3}$ imaginary, (5.16) boils down to

$$
W=\left|-\frac{1}{4} Q_{0} e^{-\chi^{0}}+\sum_{\alpha=5}^{7}\left(\frac{C_{\alpha}}{4} e^{-\chi^{\alpha}}-g_{\alpha-4} e^{\chi^{\alpha}+2 \psi}\right)+\frac{i g_{0}}{2} e^{2 \psi-\phi-\chi^{0}}\right|,
$$

where we defined $q_{0} \equiv \exp \chi^{0}$. This expression has a piece containing the gauge coupling constant $g_{0}$, which can never arise by considering the equations of motion alone (as we did in this paper), since the scalar potential is independent of $g_{0}$. On the other hand,

\footnotetext{
${ }^{15}$ The function $U$ used in [6] is related to our $\phi$ by $\phi=2 U$. Their gauge coupling constants $g_{\Lambda}$ correspond to $2 g_{I}$.
} 
in the Killing spinor equations, $g_{0}$ does appear. Ref. [6] made use of supersymmetry, which is the reason for the presence of the $g_{0}$-dependent term in (5.18). If we ignored this term, we would have

$$
W= \pm\left[\frac{1}{4} Q_{0} e^{-\chi^{0}}+\sum_{\alpha=5}^{7}\left(-\frac{C_{\alpha}}{4} e^{-\chi^{\alpha}}+g_{\alpha-4} e^{\chi^{\alpha}+2 \psi}\right)\right]
$$

which is, up to the $Q_{0}$-dependent piece and an irrelevant overall sign (that can be eliminated by taking $z \rightarrow-z$ ) exactly (4.17) with $\epsilon_{\alpha}=-1$. Our expression (4.17) is actually a reduced Hamilton's characteristic function in which the dynamics of $q_{0}$ was separated: If we consider (5.19) instead, we have (for the + sign)

$$
\dot{\chi}^{0}=4 \frac{\partial W}{\partial \chi^{0}} \quad \Rightarrow \quad \dot{q}_{0}=-Q_{0} .
$$

Comparing this with (4.10) in the limit $p \rightarrow 0$, one gets $Q_{0}=-D$. Notice that, in spite of $x^{0} \neq 0$, (4.17) has again the form (5.8), the reason for this being the decoupling of the axion $x^{0}$ from the dynamics of the other fields. This, in turn, is a consequence of the independence of the scalar potential of $g_{0}$.

\section{Conclusions and final remarks}

One of our main results is the construction of a family of nonextremal black holes for the prepotential $F=-2 i\left(X^{0} X^{1} X^{2} X^{3}\right)^{1 / 2}$, which includes both the non-BPS solutions of [14], and the supersymmetric black holes found in [5]. We discussed some of their physical properties, like entropy, temperature and several notions of mass, and showed that the product of all horizon areas depends only on the charges and the asymptotic value of the cosmological constant. We also provided a general recipe to construct non-BPS extremal solutions for an arbitrary prepotential, as long as an axion-free condition holds. These follow from a set of first-order conditions, and are related to the corresponding supersymmetric black holes by a multiplication of the charge vector with a constant field rotation matrix. We showed that the fake superpotential driving this first-order flow coincides with Hamilton's characteristic function in a Hamilton-Jacobi formalism, and reduces in the supersymmetric case to the superpotential proposed in $[6]$.

Some questions for future work are:

1. We saw in section 3.2 that the mass (3.21) correctly vanishes in the BPS case, but also for certain values of the parameters that do not correspond to any known supersymmetric solution of $\mathcal{N}=2$ gauged supergravity. It would be interesting 
to see whether they are BPS in extended supergravity theories, as conjectured in [29] for solutions of the $t^{3}$ model. In the ungauged case, this does indeed happen, and was termed 'camouflaged supersymmetry' in [31].

2. Can we generalize the fake superpotential construction of section 5 to include also axionic solutions? A hint how to do this may come from the true superpotential (5.16) that works also in the axionic case.

3. Add rotation to the static nonextremal solutions constructed here. This is under investigation.

\section{Acknowledgments}

This work was partially supported by INFN and MIUR-PRIN contract 2009-KHZKRX. The work of O.V. is supported by an STFC studentship. D.K. would like to thank Kiril Hristov for useful discussions.

\section{A. Hesse potential for $F=-2 i\left(Y^{0} Y^{1} Y^{2} Y^{3}\right)^{1 / 2}$ model}

We will consider the STU model characterised by the prepotential

$$
F=-2 i\left(Y^{0} Y^{1} Y^{2} Y^{3}\right)^{1 / 2} .
$$

The Kähler potential for this model can be written as

$$
e^{-\mathcal{K}}=8 Y^{0} \bar{Y}^{0} \operatorname{Im}\left(i \sqrt{\frac{Z^{2} Z^{3}}{Z^{1}}}\right) \operatorname{Im}\left(i \sqrt{\frac{Z^{1} Z^{3}}{Z^{2}}}\right) \operatorname{Im}\left(i \sqrt{\frac{Z^{1} Z^{2}}{Z^{3}}}\right),
$$

where $Z^{A}=X^{A} / X^{0}$. As in the main text, we define $x^{I}:=\operatorname{Re}\left(Y^{I}\right)$ and $y_{I}:=\operatorname{Re}\left(F_{I}\right)$. The Hesse potential is related to the Kähler potential by $e^{-\mathcal{K}}=-2 H$, and so in order to determine the Hesse potential we simply need to find an expression for the Kähler potential in terms of $x^{I}, y_{I}$.

By direct calculation one may show that

$$
\frac{y_{3} i \sqrt{\frac{Z^{2} Z^{3}}{Z^{1}}}-x^{2}}{x^{0} i \sqrt{\frac{Z^{2} Z^{3}}{Z^{1}}}+y_{1}}=i \sqrt{\frac{\bar{Z}^{1} \bar{Z}^{2}}{\bar{Z}^{3}}},
$$

and since the calculation is identical if we interchange $X^{2} \leftrightarrow X^{3}$ we also have

$$
\frac{y_{2} i \sqrt{\frac{Z^{2} Z^{3}}{Z^{1}}}-x^{3}}{x^{0} i \sqrt{\frac{Z^{2} Z^{3}}{Z^{1}}}+y_{1}}=i \sqrt{\frac{\bar{Z}^{1} \bar{Z}^{3}}{\bar{Z}^{2}}},
$$


Again by direct calculation one may show that

$$
\frac{x^{1} i \sqrt{\frac{Z^{2} Z^{3}}{Z^{1}}}+y_{0}}{x^{0} i \sqrt{\frac{Z^{2} Z^{3}}{Z^{1}}}+y_{1}}=\bar{Z}^{1} .
$$

Notice that (A.1) times (A.2) yields minus (A.3), and so we obtain a quadratic equation for $A:=i\left(Z^{2} Z^{3} / Z^{1}\right)^{1 / 2}$, which is given by

$$
A^{2}(\underbrace{x^{0} x^{1}+y_{2} y_{3}}_{a})+A(\underbrace{y_{0} x^{0}+y_{1} x^{1}-y_{2} x^{2}-y_{3} x^{3}}_{b})+\underbrace{y_{0} y_{1}+x^{2} x^{3}}_{c}=0 .
$$

We will only consider coordinate patches in which $4 a c>b^{2}$, and so we can write the imaginary part of the solution as

$$
\operatorname{Im}\left(i \sqrt{\frac{Z^{2} Z^{3}}{Z^{1}}}\right)= \pm \frac{\sqrt{4 a c-b^{2}}}{2\left(x^{0} x^{1}+y_{2} y_{3}\right)} .
$$

Given the symmetry of the model we can cycle through the coordinates $X^{1} \leftrightarrow X^{2} \leftrightarrow$ $X^{3}$, and so we also have

$$
\begin{aligned}
& \operatorname{Im}\left(i \sqrt{\frac{Z^{1} Z^{3}}{Z^{2}}}\right)= \pm \frac{\sqrt{4 a c-b^{2}}}{2\left(x^{0} x^{2}+y_{1} y_{3}\right)}, \\
& \operatorname{Im}\left(i \sqrt{\frac{Z^{1} Z^{2}}{Z^{3}}}\right)= \pm \frac{\sqrt{4 a c-b^{2}}}{2\left(x^{0} x^{3}+y_{1} y_{2}\right)}
\end{aligned}
$$

A separate calculation allows us to express $X^{0}$ directly in terms of $x^{I}, y_{I}$,

$$
X^{0}=\frac{x^{0} i \sqrt{\frac{Z^{2} Z^{3}}{Z^{1}}}+y_{1}}{\operatorname{Im}\left(i \sqrt{\frac{Z^{2} Z^{3}}{Z^{1}}}\right)} .
$$

Substituting (A.4), (A.5), A.6) and (A.7) into the expression for the Kähler potential we find

$$
e^{-\mathcal{K}}=4 \sqrt{4 a c-b^{2}}
$$

where we have made appropriate sign choices such that the Kähler potential is strictly positive. The Hesse potential is therefore given by

$$
\begin{array}{r}
H(x, y)=-2\left(-\left(y_{0} x^{0}-y_{1} x^{1}-y_{2} x^{2}-y_{3} x^{3}\right)^{2}+4 y_{1} x^{1} y_{2} x^{2}+4 y_{1} x^{1} y_{3} x^{3}\right. \\
\left.+4 y_{2} x^{2} y_{3} x^{3}+4 y_{0} y_{1} y_{2} y_{3}+4 x^{0} x^{1} x^{2} x^{3}\right)^{1 / 2} .
\end{array}
$$


Note that upon making the truncation

$$
\begin{array}{ll}
x^{0} \rightarrow x^{0}, & y_{0} \rightarrow y_{0}, \\
x^{1} \rightarrow x^{1}, & y_{1} \rightarrow \frac{1}{3} y_{1}, \\
x^{2} \rightarrow x^{1}, & y_{2} \rightarrow \frac{1}{3} y_{1}, \\
x^{3} \rightarrow x^{1}, & y_{3} \rightarrow \frac{1}{3} y_{1},
\end{array}
$$

one obtains the Hesse potential for the $t^{3}$ model found in [19].

We could alternatively have calculated this Hesse potential by making a symplectic rotation of the Hesse potential for $F=-X^{1} X^{2} X^{3} / X^{0}$, which was calculated in [20]. The symplectic transformation that links these two descriptions of the STU model is precisely that which sends

$$
(1, s, t, u,-s t u, t u, s u, s t)^{T} \longmapsto(1,-t u,-s u,-s t,-s t u, s, t, u)^{T}
$$

and is characterised by the symplectic matrix

$$
\mathcal{S}=\left(\begin{array}{cccc|cccc}
+1 & 0 & 0 & 0 & 0 & 0 & 0 & 0 \\
0 & 0 & 0 & 0 & 0 & -1 & 0 & 0 \\
0 & 0 & 0 & 0 & 0 & 0 & -1 & 0 \\
0 & 0 & 0 & 0 & 0 & 0 & 0 & -1 \\
\hline 0 & 0 & 0 & 0 & +1 & 0 & 0 & 0 \\
0 & +1 & 0 & 0 & 0 & 0 & 0 & 0 \\
0 & 0 & +1 & 0 & 0 & 0 & 0 & 0 \\
0 & 0 & 0 & +1 & 0 & 0 & 0 & 0
\end{array}\right) .
$$

The coordinates $q^{a}=\left(x^{I}, y_{I}\right)^{T}$ transform as a vector under symplectic transformations,

$$
\left(x^{0}, x^{1}, x^{2}, x^{3}, y_{0}, y_{1}, y_{2}, y_{3}\right)^{T} \longmapsto\left(x^{0},-y_{1},-y_{2},-y_{3}, y_{0}, x^{1}, x^{2}, x^{3}\right)^{T}
$$

The Hesse potential itself transforms as a scalar under symplectic transformations, $H(x, y) \mapsto H\left(x^{\prime}, y^{\prime}\right)$. One may check that applying this transformation to the Hesse potential for the $F=-X^{1} X^{2} X^{3} / X^{0}$ model given in [20] results precisely in the expression (A.8).

\section{References}

[1] G. Festuccia and N. Seiberg, "Rigid supersymmetric theories in curved superspace," JHEP 1106 (2011) 114 [arXiv:1105.0689 [hep-th]]. 
[2] D. Cassani, C. Klare, D. Martelli, A. Tomasiello and A. Zaffaroni, "Supersymmetry in Lorentzian curved spaces and holography," arXiv:1207.2181 [hep-th].

[3] K. Behrndt, M. Cvetič and W. A. Sabra, "Nonextreme black holes of five-dimensional $\mathcal{N}=2$ AdS supergravity," Nucl. Phys. B 553 (1999) 317 [hep-th/9810227].

[4] S. Bellucci, S. Ferrara, A. Marrani and A. Yeranyan, " $d=4$ black hole attractors in $\mathcal{N}=2$ supergravity with Fayet-Iliopoulos terms," Phys. Rev. D 77 (2008) 085027 [arXiv:0802.0141 [hep-th]].

[5] S. L. Cacciatori and D. Klemm, "Supersymmetric $\mathrm{AdS}_{4}$ black holes and attractors," JHEP 1001 (2010) 085 [arXiv:0911.4926 [hep-th]].

[6] G. Dall'Agata and A. Gnecchi, "Flow equations and attractors for black holes in $\mathcal{N}=2$ U(1) gauged supergravity," JHEP 1103 (2011) 037 [arXiv:1012.3756 [hep-th]].

[7] S. Kachru, R. Kallosh and M. Shmakova, "Generalized attractor points in gauged supergravity," Phys. Rev. D 84 (2011) 046003 [arXiv:1104.2884 [hep-th]].

[8] K. Inbasekar, P. K. Tripathy and P. K. Tripathy, "Generalized attractors in five-dimensional gauged supergravity," JHEP 1209 (2012) 003 [arXiv:1206.3887 [hep-th]].

[9] S. Ferrara, G. W. Gibbons and R. Kallosh, "Black holes and critical points in moduli space," Nucl. Phys. B 500 (1997) 75 [hep-th/9702103].

[10] S. Ferrara and A. Marrani, "On the moduli space of non-BPS attractors for $\mathcal{N}=2$ symmetric manifolds," Phys. Lett. B 652 (2007) 111 [arXiv:0706.1667 [hep-th]].

[11] S. Bellucci, S. Ferrara, A. Marrani and A. Yeranyan, "stu black holes unveiled," Entropy 10 (2008) 507 [arXiv:0807.3503 [hep-th]].

[12] L. J. Romans, "Supersymmetric, cold and lukewarm black holes in cosmological Einstein-Maxwell theory," Nucl. Phys. B 383 (1992) 395 [hep-th/9203018].

[13] M. M. Caldarelli and D. Klemm, "Supersymmetry of Anti-de Sitter black holes," Nucl. Phys. B 545 (1999) 434 [hep-th/9808097].

[14] M. J. Duff and J. T. Liu, "Anti-de Sitter black holes in gauged $\mathcal{N}=8$ supergravity," Nucl. Phys. B 554 (1999) 237 [hep-th/9901149].

[15] Z. -W. Chong, M. Cvetič, H. Lu and C. N. Pope, "Charged rotating black holes in four-dimensional gauged and ungauged supergravities," Nucl. Phys. B 717 (2005) 246 [hep-th/0411045]. 
[16] D. Klemm, "Rotating BPS black holes in matter-coupled $\mathrm{AdS}_{4}$ supergravity," JHEP 1107 (2011) 019 [arXiv:1103.4699 [hep-th]].

[17] M. Colleoni and D. Klemm, "Nut-charged black holes in matter-coupled $\mathcal{N}=2, D=4$ gauged supergravity," Phys. Rev. D 85 (2012) 126003 [arXiv:1203.6179 [hep-th]].

[18] S. L. Cacciatori, D. Klemm, D. S. Mansi and E. Zorzan, "All timelike supersymmetric solutions of $\mathcal{N}=2, D=4$ gauged supergravity coupled to abelian vector multiplets," JHEP 0805 (2008) 097 [arXiv:0804.0009 [hep-th]].

[19] D. Klemm and O. Vaughan, "Nonextremal black holes in gauged supergravity and the real formulation of special geometry," arXiv:1207.2679 [hep-th].

[20] T. Mohaupt and O. Vaughan, "The Hesse potential, the c-map and black hole solutions," JHEP 1207 (2012) 163 [arXiv:1112.2876 [hep-th]].

[21] M. Cvetič, G. W. Gibbons and C. N. Pope, "Universal area product formulae for rotating and charged black holes in four and higher dimensions," Phys. Rev. Lett. 106 (2011) 121301 [arXiv:1011.0008 [hep-th]].

[22] T. Mohaupt and K. Waite, "Instantons, black holes and harmonic functions," JHEP 0910 (2009) 058 [arXiv:0906.3451 [hep-th]].

[23] T. Mohaupt and O. Vaughan, "Non-extremal black holes, harmonic functions, and attractor equations," Class. Quant. Grav. 27 (2010) 235008 [arXiv:1006.3439 [hep-th]].

[24] T. Mohaupt and O. Vaughan, "Non-extremal black holes from the generalised r-map," arXiv:1208.4302 [hep-th].

[25] P. Meessen, T. Ortín, J. Perz and C. S. Shahbazi, "H-FGK formalism for black-hole solutions of $\mathcal{N}=2, d=4$ and $d=5$ supergravity," Phys. Lett. B 709 (2012) 260 [arXiv:1112.3332 [hep-th]].

[26] V. Cortés, "A holomorphic representation formula for parabolic hyperspheres," Proceedings of the international conference "PDEs, Submanifolds and Affine Differential Geometry" (Warsaw 2000) vol 57 ed Opozda B, Simon U and Wiehe M (Banach Center Publications (Polish Academy of Sciences, Institute of Mathematics)) pp 11-16 [math/0107037].

[27] K. Hristov, C. Toldo and S. Vandoren, "On BPS bounds in $D=4, \mathcal{N}=2$ gauged supergravity," JHEP 1112 (2011) 014 [arXiv:1110.2688 [hep-th]].

[28] K. Hristov, "On BPS bounds in $D=4, \mathcal{N}=2$ gauged supergravity II: General matter couplings and black hole masses," JHEP 1203 (2012) 095 [arXiv:1112.4289 [hep-th]]. 
[29] C. Toldo and S. Vandoren, "Static nonextremal $\mathrm{AdS}_{4}$ black hole solutions," JHEP 1209 (2012) 048 [arXiv:1207.3014 [hep-th]].

[30] R. R. Khuri and T. Ortín, "Supersymmetric black holes in $\mathcal{N}=8$ supergravity," Nucl. Phys. B 467 (1996) 355 [hep-th/9512177].

[31] I. Bena, H. Triendl and B. Vercnocke, "Camouflaged supersymmetry in solutions of extended supergravities," Phys. Rev. D 86 (2012) 061701 [arXiv:1111.2601 [hep-th]].

[32] V. Balasubramanian and P. Kraus, "A stress tensor for anti-de Sitter gravity," Commun. Math. Phys. 208 (1999) 413 [hep-th/9902121].

[33] M. Bianchi, D. Z. Freedman and K. Skenderis, "Holographic renormalization," Nucl. Phys. B 631 (2002) 159 [hep-th/0112119].

[34] L. Andrianopoli, R. D'Auria, E. Orazi and M. Trigiante, "First order description of $D=4$ static black holes and the Hamilton-Jacobi equation," Nucl. Phys. B 833 (2010) 1 [arXiv:0905.3938 [hep-th]].

[35] M. Trigiante, T. Van Riet and B. Vercnocke, "Fake supersymmetry versus Hamilton-Jacobi," JHEP 1205 (2012) 078 [arXiv:1203.3194 [hep-th]].

[36] A. Ceresole and G. Dall'Agata, "Flow equations for non-BPS extremal black holes," JHEP 0703 (2007) 110 [hep-th/0702088].

[37] G. Lopes Cardoso, A. Ceresole, G. Dall'Agata, J. M. Oberreuter and J. Perz, "First-order flow equations for extremal black holes in very special geometry," JHEP 0710 (2007) 063 [arXiv:0706.3373 [hep-th]].

[38] T. Ortín, "Extremality versus supersymmetry in stringy black holes," Phys. Lett. B 422 (1998) 93 [hep-th/9612142].

[39] S. Barisch, G. Lopes Cardoso, M. Haack, S. Nampuri and N. A. Obers, "Nernst branes in gauged supergravity," JHEP 1111 (2011) 090 [arXiv:1108.0296 [hep-th]].

[40] G. L. Cardoso and V. Grass, "On five-dimensional non-extremal charged black holes and FRW cosmology," Nucl. Phys. B 803 (2008) 209 [arXiv:0803.2819 [hep-th]].

[41] S. Barisch-Dick, G. L. Cardoso, M. Haack and S. Nampuri, "Extremal black brane solutions in five-dimensional gauged supergravity," arXiv:1211.0832 [hep-th]. 